\title{
Inhibitory Actions of Tropeines on the $\alpha 3$ Glycine Receptor Function
}

\author{
Victoria P. San Martín, Carlos F. Burgos, Ana M. Marileo, Cesar O. Lara, Anggelo Sazo, \\ Jorge Fuentealba, Leonardo Guzmán, Patricio A. Castro, Luis G. Aguayo, \\ Gustavo Moraga-Cid* and Gonzalo E. Yévenes*
}

Department of Physiology, Faculty of Biological Sciences, University of Concepción, Concepción, Chile

\section{OPEN ACCESS}

Edited by:

Milica S. Prostran,

University of Belgrade, Serbia

Reviewed by:

Gillian Grafton,

University of Birmingham,

United Kingdom

Thomas Heinbockel,

Howard University, United States

*Correspondence:

Gustavo Moraga-Cid

gumoraga@udec.cl

Gonzalo E. Yévenes

gyevenes@udec.cl

Specialty section:

This article was submitted to

Neuropharmacology,

a section of the journal

Frontiers in Pharmacology

Received: 16 October 2018

Accepted: 19 March 2019

Published: 08 April 2019

Citation:

San Martín VP, Burgos CF Marileo AM, Lara CO, Sazo A, Fuentealba J, Guzmán L, Castro PA,

Aguayo LG, Moraga-Cid G and Yévenes GE (2019) Inhibitory Actions

of Tropeines on the $\alpha 3$ Glycine

Receptor Function.

Front. Pharmacol. 10:331. doi: 10.3389/fphar.2019.00331
Glycine receptors (GlyRs) are chloride-permeable pentameric ligand-gated ion channels. The inhibitory activity of GlyRs is essential for many physiological processes, such as motor control and respiration. In addition, several pathological states, such as hyperekplexia, epilepsy, and chronic pain, are associated with abnormal glycinergic inhibition. Recent studies have pointed out that positive allosteric modulators targeting the GlyR $\alpha 3$ subunit ( $\alpha 3$ GlyR) displayed beneficial effects in chronic pain models. Interestingly, previous electrophysiological studies have shown that tropeines, which are a family of synthetic antagonists of the serotonin type 3 receptors $\left(5-\mathrm{HT}_{3} \mathrm{Rs}\right)$, potentiate the activity of GlyRs conformed by $\alpha 1$ subunits. However, despite its importance as a pharmacological target in chronic pain, it is currently unknown whether the a3GlyR function is modulated by tropeines. Using electrophysiological techniques and molecular docking simulations, here we show that tropeines are inhibitors of the $\alpha 3$ GlyR function. Tropisetron, a prototypical tropeine, exerted concentration-dependent inhibitory effects on $\alpha 3$ GlyRs at the low micromolar range. In addition, three other tropeines showed similar effects. Single-channel recordings show that tropisetron inhibition is associated with a decrease in the open probability of the ion channel. Molecular docking assays suggest that tropeines preferentially bind to an agonist-free, closed state of the ion channel. The tropeine binding occurs in a discrete pocket around the vicinity of the orthosteric site within the extracellular domain of a3GlyR. Thus, our results describe the pharmacological modulation of tropeines on $\alpha 3$ GlyRs. These findings may contribute to the development of GlyR-selective tropeine derivatives for basic and/or clinical applications.

Keywords: glycine receptor, tropeines, modulation, ligand-gated ion channels, electrophysiology, molecular docking

\section{INTRODUCTION}

Strychnine-sensitive glycine receptors (GlyRs) are anion-selective neurotransmitter-gated inhibitory ion channels. GlyRs belong to the pentameric ligand-gated ion channel superfamily, together with the inhibitory $\mathrm{GABA}_{\mathrm{A}}$ receptor and the excitatory nicotinic acetylcholine receptor (nAchR) and serotonin type 3 receptors (5-HT3Rs) (Lynch, 2004; Lynch, 2009; Zeilhofer et al., 2012). In the mammalian CNS, the enhancement of the chloride conductance through GlyR activation results in a transient hyperpolarization of the membrane potential, which contributes to the control of neuronal excitability. The GlyR-mediated inhibition controls critical 
neurophysiological functions, such as motor coordination, respiratory control, muscle tone, as well as pain processing. Interestingly, several pathological states, such as hyperekplexia, epilepsy, autism, and chronic pain, have been associated with alterations on glycinergic inhibition (Harvey et al., 2004, 2008; Eichler et al., 2008; Pilorge et al., 2016).

Glycine receptors are pentameric complexes composed of $\alpha$ and $\beta$ subunits, which can form receptors composed exclusively of $\alpha$ subunits (i.e., homomeric) or of $\alpha$ and $\beta$ subunits (i.e., heteromeric) (Lynch, 2004; Corringer et al., 2012). Up until now, four $\alpha$ subunits $(\alpha 1-4)$ and a single $\beta$ subunit have been described. Although the $\alpha$ subunits share a high degree of sequence homology, they also exhibit important differences in their biophysical and pharmacological properties as well as in their expression patterns. These specific features are possibly linked to their proposed roles in physiological and pathological states (Lynch, 2009). A single GlyR subunit possesses an extracellular domain (ECD), four transmembrane domains (TM1-4) and an intracellular domain between the TM3 and TM4 domains (ICD). The binding of the neurotransmitter glycine to the ECD triggers the process of receptor activation, which results in the opening of the ion channel pore.

Despite the importance of GlyRs in physiological processes and in disease states, the present state of GlyR pharmacology is still limited (Yévenes and Zeilhofer, 2011; Zeilhofer et al., 2018). To date, only few agonists and antagonists of GlyRs have been characterized. Fortunately, recent studies have significantly expanded the GlyR pharmacology with the characterization of novel glycinergic positive allosteric modulators with analgesic actions in behavioral models of chronic pain (Zeilhofer et al., 2018). These studies pointed out that the potentiation of GlyRs composed of the $\alpha 3$ subunit is required for their in vivo analgesic actions. In this context, previous studies have shown that low nanomolar concentrations of several tropeines, which are a family of synthetic antagonists of the $5-\mathrm{HT}_{3} \mathrm{Rs}$ (Haus et al., 2004), potentiate GlyRs composed of $\alpha 1$ subunits (Chesnoy-Marchais, 1996; Supplisson and Chesnoy-Marchais, 2000; Yang et al., 2007; Maksay et al., 2009). However, despite its importance as a pharmacological target in chronic pain, it is currently unknown whether the $\alpha 3$ GlyR function is modulated by tropeines in a similar fashion. Defining the actions of this class of ligands on $\alpha 3$ GlyRs will elucidate their pharmacological potential as chemical templates for the development of novel glycinergic modulators. Using electrophysiological techniques and molecular docking simulations, here we show that tropeines exerted inhibitory actions on $\alpha 3$ GlyRs. In addition, our molecular docking studies suggest that tropeines likely bind to a discrete site near the glycine-binding site, preferentially in an agonist-free closed state of the ion channel.

\section{MATERIALS AND METHODS}

\section{Cell Culture and Transfection}

HEK 293 cells (CRL-1573; American Type Culture Collection, Manassas, VA, United States) were cultured using standard methods (Lara et al., 2016). The cells were transfected using
XfectTM Transfection Reagent (Clontech, United States) using $1.0 \mu \mathrm{g}$ of cDNA plasmid encoding the $\alpha 3 \mathrm{GlyR}$ subunit and $0.5 \mu \mathrm{g}$ of EGFP. All recordings were made $24-36 \mathrm{~h}$ after transfection.

\section{Electrophysiology}

Glycine-evoked currents were recorded from transfected HEK 293 cells in the whole-cell voltage-clamp configuration at room temperature $\left(20-24^{\circ} \mathrm{C}\right.$ ) at a holding potential of $-60 \mathrm{mV}$ (Lara et al., 2016). Patch electrodes (3-4 $\mathrm{m} \Omega$ ) were pulled from borosilicate glass and were filled with (in $\mathrm{mM}$ ): $120 \mathrm{CsCl}, 8$ EGTA, 10 HEPES (pH 7.4), $4 \mathrm{MgCl}_{2}, 0.5$ GTP and 2 ATP. The external solution contained (in $\mathrm{mM}$ ) $140 \mathrm{NaCl}, 5.4 \mathrm{KCl}$, $2.0 \mathrm{CaCl}_{2}, 1.0 \mathrm{MgCl}_{2}, 10 \mathrm{HEPES}$ ( $\mathrm{pH} 7.4$ ), and 10 glucose. Whole-cell recordings were performed with an Axoclamp 200B amplifier (Molecular Devices, United States) and acquired using Clampex 10.1 software. Data analysis was performed off-line using Clampfit 10.1 (Axon Instruments, Sunnyvale, CA, United States). Exogenous glycine-evoked currents were obtained using a manually applied pulse (3-4 s) of the agonist and an outlet tube (200 $\mu \mathrm{m}$ ID) of a custom-designed gravity-fed microperfusion system (flow rate $\approx 0.3-0.4 \mathrm{ml} / \mathrm{min}$; exchange rate $\approx 50-80 \mathrm{~ms}$ ) positioned $50-100 \mu \mathrm{m}$ from the recorded cell. The methodologies employed for the single channel recordings of $\alpha 3$ GlyRs in cell-attached configuration have been previously published (Marabelli et al., 2013; Lara et al., 2016). The patch pipettes (non-sylgard-coated) had tip resistances of $10-20 \mathrm{~m} \Omega$ and were manually fire polished in a microforge (Narishige, Japan). The data were filtered (1-kHz low-pass 8-pole Butterworth) and acquired at $5-20 \mathrm{kHz}$ using an Axopatch 200B amplifier and a 1322A Digidata (Axon Instruments, Union City, CA, United States). Data was acquired using pClamp software and analyzed off-line with Clampfit 10.1 (Axon Instruments, Union City, CA, United States). Tropeine stocks were prepared in high purity distilled water and subsequently diluted into the recording solution on the day of the experiment. Tropeines were obtained from AK Science (CA, United States). All other reagents were from Sigma-Aldrich (St. Louis, MO, United States).

\section{Molecular Docking Procedures}

Protein-ligand docking was performed using the structures of $\alpha 3$ and $\alpha 1$ GlyRs obtained from the Protein DataBank (PDB ID: 5CFB, 5TIO, 3JAD, and 3JAE) (Du et al., 2015; Huang et al., 2015). The structures of tropisetron, granisetron, dolasetron, and ondansetron are available in the PubChem database (CID: 656665, 3510, 3033818, and 4595) and were prepared using LigPrep before docking simulations. All complexes were created with Glide (Schrödinger, LLC, New York, NY, 2016) using a receptor grid centered on the amino acids that form the orthosteric binding site for glycine [(+)F159,Y202,T204,F207, (-)R65,S129, on $\alpha 3 \mathrm{GlyR}]$, and an extra-precision (XP) configuration. Analysis of the interface GlyR-tropeines included structural and energetic parameters performed by the same software. To estimate ligand-binding affinity, a theoretical Gibbs free energy of binding, $\Delta \mathrm{G}_{\text {bind }}$, was calculated by an energy calculation MM-GBSA using Prime (Schrödinger, LLC, New York, NY, 2016). All images were created using PyMol (v1.5, DeLano Scientific LLC, United States). 
A

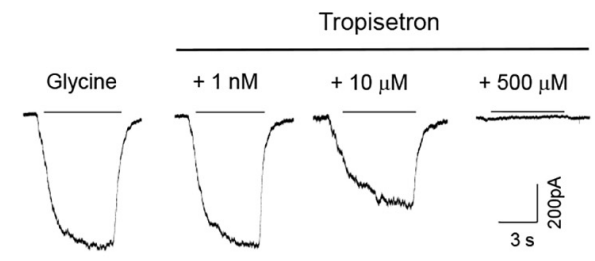

B

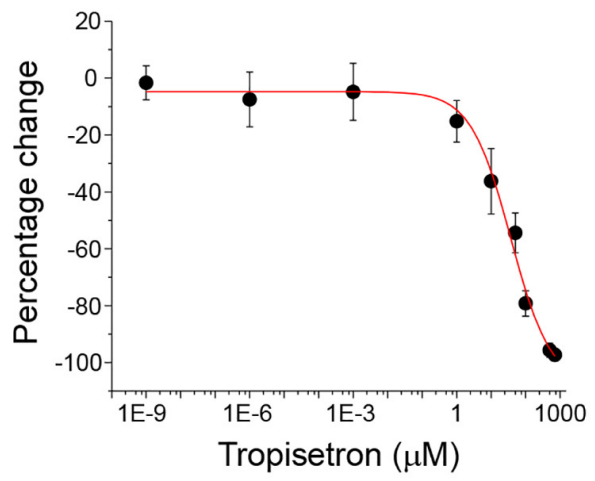

E

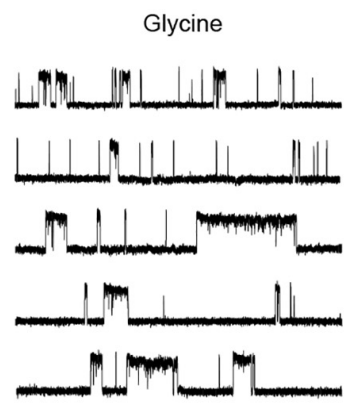

C

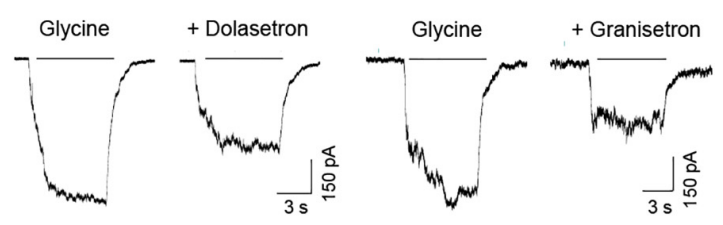

D

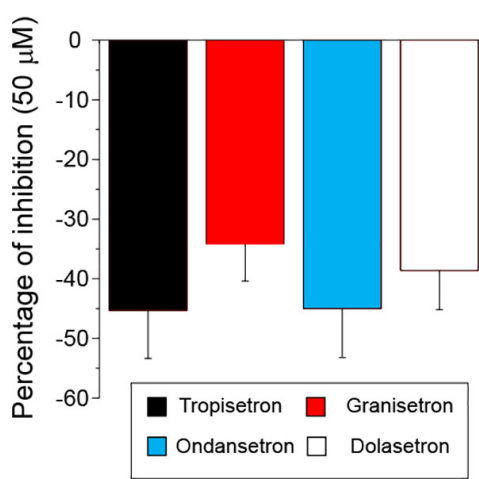

$\mathbf{F}$

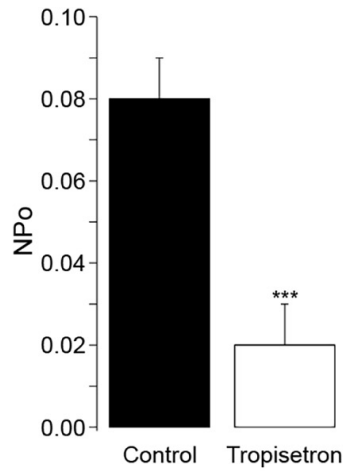

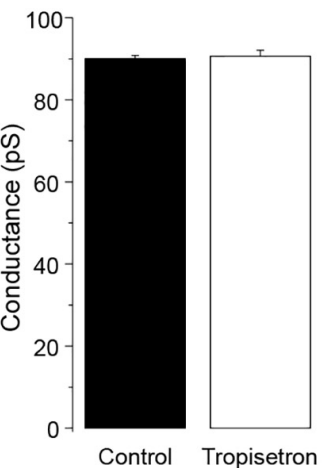

FIGURE 1 | Functional modulation of homomeric $\alpha 3$ GlyRs by tropeines. (A) Whole-cell current traces evoked by 30-60 $\mu \mathrm{M}$ glycine before and during the application of tropisetron ( $1 \mathrm{nM}, 10 \mu \mathrm{M}$, and $500 \mu \mathrm{M}$ ) from a single HEK293 cell expressing a3GlyRs. (B) The graph summarizes the effect of different tropisetron concentrations ( $1 \mathrm{fM}-700 \mu \mathrm{M}$ ) on the glycine-activated currents $(n=8)$. (C) Current traces evoked by 30-60 $\mu \mathrm{M}$ glycine before and after the application of $50 \mu \mathrm{M}$ granisetron and dolasetron from two different HEK293 cells expressing $\alpha 3$ GlyRs (D) The plot summarizes the effects of $50 \mu \mathrm{M}$ granisetron ( $n=6$ ), ondansetron $(n=8)$, dolasetron $(n=7)$ and tropisetron $(n=6)$ on the glycine-activated currents of $\alpha 3$ GlyRs. Differences were not significant (ANOVA followed by Bonferroni post hoc test, $F(3,26)=0.50$ ). (E) Single-channel recordings in the cell-attached configuration from a cell expressing $\alpha 3 G$ lyRs before and in the presence of $50 \mu \mathrm{M}$ of tropisetron. (F) The graphs show that tropisetron significantly decreased the open probability of a3GlyRs, but did not modify the main conductance $(* * * P<0.001$, paired Student $t$-test; $n=4)$.

\section{Data Analysis}

All values were expressed as mean \pm s.e.m of normalized agonist-activated currents. $P<0.05$ was considered statistically significant. Multiple comparisons were analyzed with ANOVA followed by a Bonferroni post hoc test. All the statistical analyses and plots were performed with MicroCal Origin 8.0 (Northampton, MA, United States).

\section{RESULTS}

We first examined the sensitivity of the homomeric $\alpha 3$ GlyR to different concentrations of tropisetron (also known as ICS-205,930), a prototypical tropeine which is widely used as an anti-emetic drug. Application of tropisetron alone to cells expressing $\alpha 3$ GlyRs did not elicit any detectable change in the holding currents, suggesting the absence of agonistic activity (not shown). Using a sub-saturating concentration of glycine $\left(\mathrm{EC}_{5-10}\right)$ to activate GlyRs, we found that nanomolar concentrations of tropisetron did not modify the amplitude of the chloride currents through $\alpha 3$ GlyRs (Figure 1A). On the other hand, concentrations of tropisetron above $1 \mu \mathrm{M}$ exerted a significant inhibitory effect on the $\alpha 3 \mathrm{GlyR}$ activity (Figures 1A,B). The concentration-response curve analysis displayed an $\mathrm{IC}_{50}$ of $37 \pm 11 \mu \mathrm{M}(n=8)$, with a maximal inhibition of $-97.2 \pm 2.1 \%$ with $700 \mu \mathrm{M}$ of tropisetron $(n=8)$. To obtain additional insights on the chemical determinants associated with the inhibition of 
A

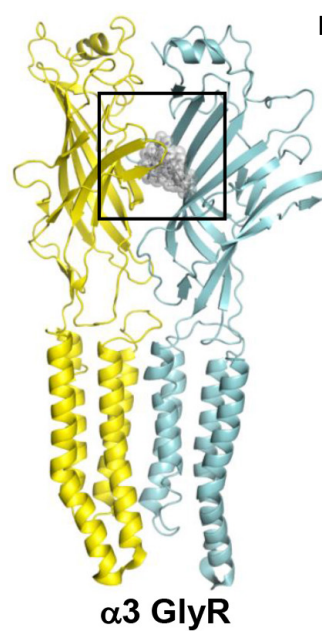

B

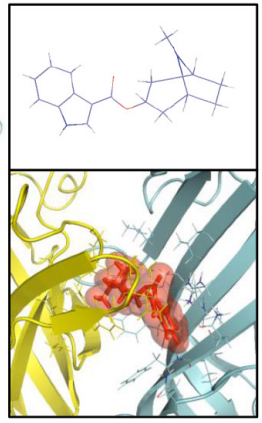

TROPISETRON

DS $\quad-9.475$

$\Delta \mathbf{G}_{\text {bind }}-58.74$

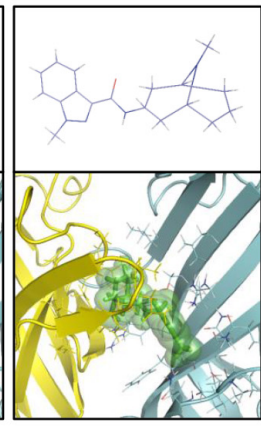

GRANISETRON

$-8.572$

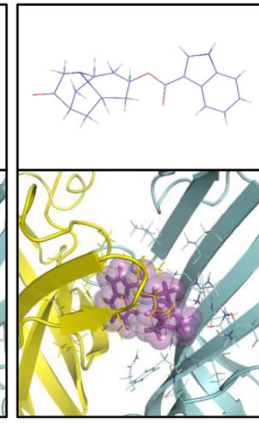

DOLASETRON $-6.064$ $-46.14$

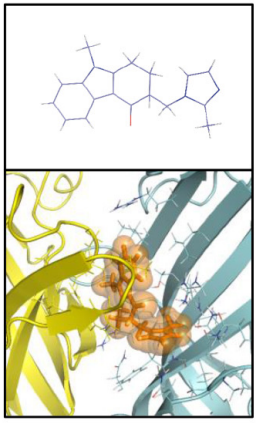

ONDANSETRON $-4.824$ $-54.91$

C
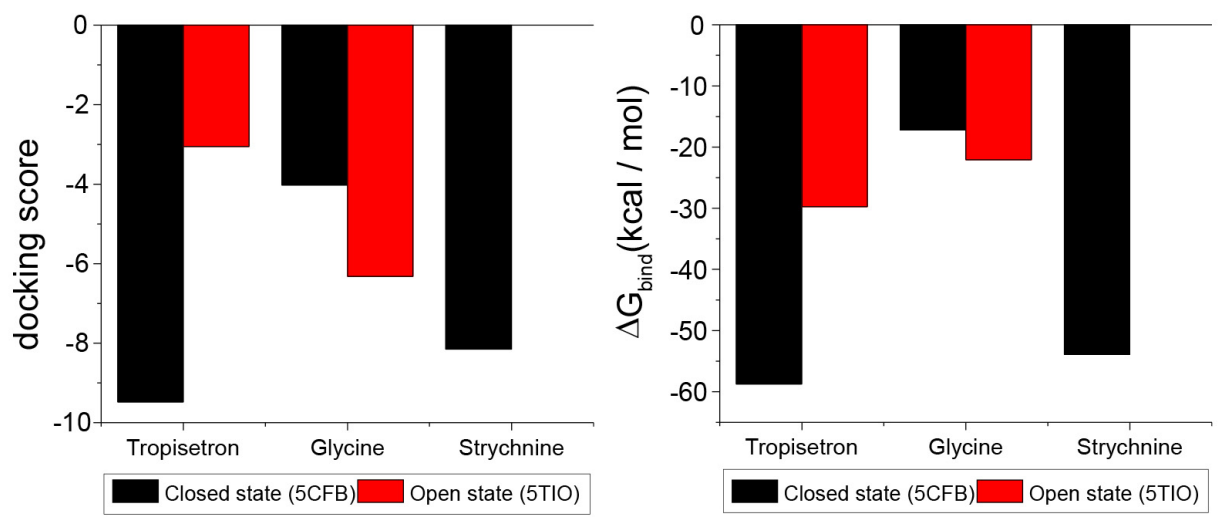

FIGURE 2 | Putative binding sites of tropeines on the extracellular domain of $\alpha 3$ GlyRs. (A) The $\alpha 3 / \alpha 3$ dimer in closed conformation (from the 5 CFB structure) shows the predicted binding site of the tropeines experimentally tested (gray spheres). Both protein chains are identical and were colored in cyan and yellow. (B) 2D structures (upper panels) and binding site on $\alpha 3 G$ GlyR for tropisetron, granisetron, dolasetron, and ondansetron (lower panels). For all complexes, the side chains of the amino acids at $5 \AA$ around the modulator and forming part of the binding site are shown. Docking score and $\Delta G_{\text {bind }}$ values are presented for each compound. (C) The graphs compare the docking scores and the theoretical $\Delta G_{\text {bind }}$ of tropisetron interaction with a $3 G$ lyRs in the closed and in the open state. The values obtained from the docking procedures with glycine and strychnine under similar conditions has been added as references.

$\alpha 3$ GlyR by tropeines, we next analyzed the effects of three other tropeines on the $\alpha 3$ GlyR function. We found that the application of $50 \mu \mathrm{M}$ of granisetron, ondansetron, and dolasetron exerted a significant inhibition of the glycineactivated currents (Figures 1C,D). The extent of inhibition elicited by these compounds was not significantly different to those obtained with an equivalent concentration of tropisetron (Figure 1D), suggesting a conserved mechanism of inhibition. To investigate further the mechanism underlying the ion channel modulation, we next studied the tropeine modulation of $\alpha 3$ GlyRs by performing single-channel recordings in the cell-attached configuration (Figures 1E,F). In agreement with our data obtained using whole-cell currents, the application of $50 \mu \mathrm{M}$ of tropisetron to membrane patches expressing $\alpha 3$ GlyRs significantly decreased the normalized open probability $(\mathrm{nPo})$ by $-76 \pm 5.5 \%$. On the other hand, tropeine did not elicit any significant change in the ion channel mean amplitude (Control $=5.40 \pm 0.05 \mathrm{pA}$ vs. tropisetron $=5.43 \pm 0.09 \mathrm{pA}, p=0.65$, paired $t$-test $)$ or main conductance (Control $=90.0 \pm 0.7 \mathrm{pS}$ vs. tropisetron $=90.6 \pm 1.5 \mathrm{pS}, p=0.59$, paired $t$-test). Collectively, these results are consistent with a mechanism of ion channel inhibition resulting from the direct binding of tropisetron to the a3GlyR structure.

In order to explore the molecular and structural determinants of the tropeine inhibition, we next performed molecular docking assays using the crystal structures of $\alpha 3$ GlyR as templates (Huang et al., 2015, 2017). All the tropeines tested were able to interact with the receptor in a favorable and stable manner (Figures 2A,B). A more detailed analysis of the binding site for tropeines showed that the amino acids S129, T204, and N42 of $\alpha 3$ GlyR can form H-bonds with the modulators, while R65, F159, and F207 generate pi-cation interactions, and E157 forms a salt bridge with tropisetron (Supplementary Figure S1). Interestingly, the comparison between closed and open states suggests a significant preference of tropisetron to bind to the 
closed conformation of the ion channel (Docking scores, closed state: -9.475; open state: -3.055) (Figures 2B,C). In addition, an increase in the theoretical $\Delta \mathrm{G}_{\mathrm{bind}}$ was predicted for the open state complex ( -58.74 to $-29.75 \mathrm{kcal} / \mathrm{mol}$ ) (Figures 2 B,C). The results obtained with tropisetron were similar for the other tropeines examined (Supplementary Table S1).

\section{DISCUSSION}

The traditional view of GlyR pharmacology comprises a limited number of agonists, antagonists and allosteric modulators (Yévenes and Zeilhofer, 2011; Zeilhofer et al., 2018). An important part of these investigations centered their attention on the $\alpha 1$ subunit of GlyRs, which is widely expressed in the mammalian spinal cord and brainstem. However, due to its relevance in chronic inflammatory pain, the $\alpha 3 \mathrm{GlyR}$ has attracted recent attention as a target for the development of novel analgesics. Several studies have demonstrated that novel compounds such as the propofol analog 2,6-di-tertbutyl phenol (2,6-DTBP), the phytocannabinoid cannabidiol (CBD), the natural alkaloid gelsemine, and the synthetic tricyclic sulfonamide AM-1488 are able to modulate glycineactivated currents through $\alpha 3$ GlyRs (Xiong et al., 2012; Acuña et al., 2016; Lara et al., 2016; Huang et al., 2017; Zeilhofer et al., 2018). Interestingly, although none of these modulators possesses a significant GlyR subunit-selectivity, they were able to reduce chronic pain symptoms in behavioral models. The tropeines examined in our study, similar to many of these modulators, have also been characterized as modulators of other GlyR subunits. Several electrophysiological studies showed that tropisetron potentiated $\alpha 1$ GlyR currents at nanomolar concentrations, while higher micromolar concentrations produced inhibition (Chesnoy-Marchais, 1996; Supplisson and Chesnoy-Marchais, 2000; Yang et al., 2007; Maksay et al., 2009). On the other hand, tropisetron only showed inhibitory effects on $\alpha 2$ GlyR activity (Supplisson and Chesnoy-Marchais, 2000). The results of the present work with $\alpha 3$ GlyRs are similar to those obtained in studies with $\alpha 2$ GlyRs and suggest that tropeines have a conserved mechanism of action on $\alpha 2$ and $\alpha 3$ GlyRs. Interestingly, additional molecular modeling and docking simulations showed that the putative binding site of tropisetron is highly conserved between $\alpha 1$ and $\alpha 3$ GlyR subunits (Supplementary Figure S2), suggesting a similar drug-receptor interaction at least in a certain concentration range. Considering all of the above, it appears that the mechanisms underlying the divergence on the functional modulation elicited by tropisetron in $\alpha 1$ GlyRs versus $\alpha 2 / \alpha 3$ GlyRs are linked to the allosteric transitions associated to the ion channel gating rather than differential binding modes of tropeines. Although the presence of more than one binding site of tropeines to GlyRs cannot be ruled out without further experimental evidence, we speculate that some GlyR configurations with very similar tropeine binding modes may trigger differential allosteric transitions, favoring a tropeine-dependent potentiation or a tropeine inhibition. Future studies may help to define a more comprehensive view of the mechanisms underlying the differential effects of tropeines on GlyRs of diverse composition.

\section{CONCLUSION}

The present work describes the sensitivity of homomeric $\alpha 3$ GlyRs to tropeines. Our results provide additional insights on the molecular mechanisms associated with the modulation of GlyRs by tropeines. A better understanding of the structural basis involved in the tropeine-GlyR interactions may contribute to the design of novel tropeine-based derivatives for basic and/or clinical applications.

\section{AUTHOR CONTRIBUTIONS}

VSM, CB, CL, AM, AS, and GM-C performed the research. JF, LG, PC, LA, GM-C, and GY designed the research and contributed with analytical tools. VSM, CB, CL, AM, GM-C, and GY analyzed the data. GM-C and GY wrote the manuscript. All authors read and approved the final version of the manuscript.

\section{FUNDING}

This work was supported by grant FONDECYT 1170252 (to GY), FONDECYT 1160851 (to GM-C), and FONDECYT 3170108 (to $\mathrm{CB}$ ). CL and AM were supported by CONICYT doctoral fellowships 21171549 and 21181287, respectively. VSM and AS were supported by the University of Concepción postgraduate fellowships (Ph.D. Program in Biological Sciences and Master Program in Neurobiology, respectively).

\section{ACKNOWLEDGMENTS}

The authors thank L. Aguayo, I. Cid, and J. Gavilán for their outstanding technical assistance.

\section{SUPPLEMENTARY MATERIAL}

The Supplementary Material for this article can be found online at: https://www.frontiersin.org/articles/10.3389/fphar.2019. 00331/full\#supplementary-material

FIGURE S1 | Ligand interaction diagrams of tropeines with the extracellular domain of $\alpha 3$ GlyR. A detailed analysis of the amino acids involved in the formation and stabilization of $\alpha 3$ GlyR complexes with (A) tropisetron, (B) granisetron, (C) dolasetron, and (D) ondansetron. All detected interactions are described in the inner box. All detected interactions between $\alpha 3 G$ GlyR and each molecule are described in the inner box with a cutoff of $4 \AA$ from the receptor. Hydrogen bonds are partially electrostatic interactions between a hydrogen atom bound to an electronegative group and an atom or a group of atoms, which acts an acceptor. In the tropeine- $\alpha 3$ GlyR interaction, S129, T204 act as hydrogen-bond donors, while the N42 acts as an acceptor. Pi-cation interactions are non-covalent interactions that occurs between a charged group with an electron-rich $\pi$ group, like aromatic rings. Pi-cation interactions were obserbed between R65, F159, and 
F207 with tropisetron and granisetron. A salt bridge combines hydrogen bonds and ionic pairing. A salt bridge between the negative charged side chain of E157 with the $\mathrm{NH} 2+$ of several tropeines was observed. All analysis was performed using Maestro (Schrödinger, LLC, New York, NY, 2016).

FIGURE S2 | Putative binding sites of tropeines on the extracellular domain of $\alpha 1$ GlyRs. (A) The $\alpha 1 / \alpha 1$ dimer in closed conformation (from the 3JAD structure) shows the predicted binding site for all tropeines tested (in gray spheres). Both chains are identical and were colored in light blue and pale yellow for representative purposes (B) 2D structure and binding sites of tropisetron, granisetron, dolasetron and ondansetron on $\alpha 1 \mathrm{GlyR}$. For all complexes, the side chains of the amino acids at $5 \AA$ around the modulator

\section{REFERENCES}

Acuña, M. A., Yévenes, G. E., Ralvenius, W. T., Benke, D., Di Lio, A., Lara, C. O., et al. (2016). Phosphorylation state-dependent modulation of spinal glycine receptors alleviates inflammatory pain. J. Clin. Invest. 126, 2547-2560. doi: 10.1172/JCI83817

Chesnoy-Marchais, D. (1996). Potentiation of chloride responses to glycine by three 5-HT3 antagonists in rat spinal neurones. Br. J. Pharmacol. 118, 2115-2125. doi: 10.1111/j.1476-5381.1996.tb15651.x

Corringer, P. J., Poitevin, F., Prevost, M. S., Sauguet, L., Delarue, M., and Changeux, J. P. (2012). Structure and pharmacology of pentameric receptor channels: from bacteria to brain. Structure 20, 941-956. doi: 10.1016/j.str.2012.05.003

Du, J., Lü, W., Wu, S., Cheng, Y., and Gouaux, E. (2015). Glycine receptor mechanism elucidated by electron cryo-microscopy. Nature 526, 224-229. doi: 10.1038 /nature 14853

Eichler, S. A., Kirischuk, S., Jüttner, R., Schafermeier, P. K., Legendre, P., Lehmann, T. N., et al. (2008). Glycinergic tonic inhibition of hippocampal neurons with depolarizing GABAergic transmission elicits histopathological signs of temporal lobe epilepsy. J. Cell. Mol. Med. 12, 2848-2866. doi: 10.1111/j.15824934.2008.00357.x

Harvey, R. J., Depner, U. B., Wässle, H., Ahmadi, S., Heindl, C., Reinold, H., et al. (2004). GlyR $\alpha 3$ : an essential target for spinal PGE2-mediated inflammatory pain sensitization. Science 304, 884-887. doi: 10.1126/science.1094925

Harvey, R. J., Topf, M., Harvey, K., and Rees, M. I. (2008). The genetics of hyperekplexia: more than startle! Trends Genet. 24, 439-447. doi: 10.1016/j.tig. 2008.06.005

Haus, U., Späth, M., and Färber, L. (2004). Spectrum of use and tolerability of 5HT3 receptor antagonists. Scand. J. Rheumatol. Suppl. 199, 12-18. doi: 10.1080/ 03009740410006961

Huang, X., Chen, H., Michelsen, K., Schneider, S., and Shaffer, P. L. (2015). Crystal structure of human glycine receptor-alpha3 bound to antagonist strychnine. Nature 526, 277-280. doi: 10.1038/nature14972

Huang, X., Shaffer, P. L., Ayube, S., Bregman, H., Chen, H., Lehto, S. G., et al. (2017). Crystal structures of human glycine receptor $\alpha 3$ bound to a novel class of analgesic potentiators. Nat. Struct. Mol. Biol. 24:108. doi: 10.1038/nsmb.3329

Lara, C. O., Murath, P., Muñoz, B., Marileo, A. M., San Martín, L., San Martín, V. P., et al. (2016). Functional modulation of glycine receptors by the alkaloid gelsemine. Br. J. Pharmacol. 173, 2263-2277. doi: 10.1111/bph.13507

Lynch, J. W. (2004). Molecular structure and function of the glycine receptor chloride channel. Physiol. Rev. 84, 1051-1095. doi: 10.1152/physrev.00042.2003

Lynch, J. W. (2009). Native glycine receptor subtypes and their physiological roles. Neuropharmacology 56, 303-309. doi: 10.1016/j.neuropharm.2008.07.034 and forming part of the binding site are shown. Docking score and $\Delta \mathrm{G}_{\text {bind }}$ are presented for each compound. $\Delta G_{b i n d}$ values were calculated using the equation $\mathrm{G}=\mathrm{E}_{\text {bond }}+\mathrm{E}_{\text {electrostic }}+\mathrm{E}_{v d w}+\mathrm{G}_{p o l}+\mathrm{G}_{n p}-\mathrm{TS}$, where $\mathrm{G}_{p o l}$ corresponds to the solvation free energy obtained by using the generalized Born (GB) model, and Gnp is an estimation from solvent accessible surface area (SASA).

TABLE S1 | Characterization of the complexes generated between the tropeines and $\alpha 3$ GlyRs in closed / open state. Docking score and $\Delta G_{\text {bind }}$ calculated for complexes generated using the 5CFB (closed) and 5TIO (open) structures of $\alpha 3 G l y R$. Values for glycine and strychnine obtained using a protocol similar to tropeines have been added as references.

Maksay, G., Laube, B., Schemm, R., Grudzinska, J., Drwal, M., and Betz, H. (2009). Different binding modes of tropeines mediating inhibition and potentiation of $\alpha 1$ glycine receptors. J. Neurochem. 109, 1725-1732. doi: 10.1111/j.1471-4159. 2009.06083.x

Marabelli, A., Moroni, M., Lape, R., and Sivilotti, L. G. (2013). The kinetic properties of the $\alpha 3$ rat glycine receptor make it suitable for mediating fast synaptic inhibition. J. Physiol. 591, 3289-3308. doi: 10.1113/jphysiol.2013. 252189

Pilorge, M., Fassier, C., Le Corronc, H., Potey, A., Bai, J., De Gois, S., et al. (2016). Genetic and functional analyses demonstrate a role for abnormal glycinergic signaling in autism. Mol. Psychiatry 21:936. doi: 10.1038/mp.2015.139

Supplisson, S., and Chesnoy-Marchais, D. (2000). Glycine receptor $\beta$ subunits play a critical role in potentiation of glycine responses by ICS-205,930. Mol. Pharmacol. 58, 763-770. doi: 10.1124/mol.58.4.763

Xiong, W., Cui, T., Cheng, K., Yang, F., Chen, S. R., Willenbring, D., et al. (2012). Cannabinoids suppress inflammatory and neuropathic pain by targeting $\alpha 3$ glycine receptors. J. Exp. Med. 209, 1121-1134. doi: 10.1084/jem.20120242

Yang, Z., Ney, A., Cromer, B. A., Ng, H. L., Parker, M. W., and Lynch, J. W. (2007). Tropisetron modulation of the glycine receptor: femtomolar potentiation and a molecular determinant of inhibition. J. Neurochem. 100, 758-769. doi: 10.1111/ j.1471-4159.2006.04242.x-

Yévenes, G. E., and Zeilhofer, H. U. (2011). Allosteric modulation of glycine receptors. Br. J. Pharmacol. 164, 224-236. doi: 10.1111/j.1476-5381.2011.01471.x

Zeilhofer, H. U., Acuña, M. A., Gingras, J., and Yévenes, G. E. (2018). Glycine receptors and glycine transporters: targets for novel analgesics? Cell. Mol. Life Sci. 75, 447-465. doi: 10.1007/s00018-017-2622-x

Zeilhofer, H. U., Wildner, H., and Yévenes, G. E. (2012). Fast synaptic inhibition in spinal sensory processing and pain control. Physiol. Rev. 92, 193-235. doi: 10.1152 /physrev.00043.2010

Conflict of Interest Statement: The authors declare that the research was conducted in the absence of any commercial or financial relationships that could be construed as a potential conflict of interest.

Copyright (C) 2019 San Martín, Burgos, Marileo, Lara, Sazo, Fuentealba, Guzmán, Castro, Aguayo, Moraga-Cid and Yévenes. This is an open-access article distributed under the terms of the Creative Commons Attribution License (CC BY). The use, distribution or reproduction in other forums is permitted, provided the original author(s) and the copyright owner(s) are credited and that the original publication in this journal is cited, in accordance with accepted academic practice. No use, distribution or reproduction is permitted which does not comply with these terms. 